\title{
Clinical factors in patients with congenital muscular torticollis treated with surgical resection
}

\author{
Sue Min Kim ${ }^{1}$, Bohwan $\mathrm{Cha}^{2}$, Kwang Sik Jeong ${ }^{2}$, Non Hyeon $\mathrm{Ha}^{2}$, Myong Chul Park ${ }^{2}$ \\ ${ }^{1}$ Department of Plastic and Reconstructive Surgery, National Medical Center, Seoul; ${ }^{2}$ Department of Plastic and Reconstructive Surgery, Ajou \\ University Hospital, Suwon, Korea
}

Background Congenital muscular torticollis (CMT) is characterized by persistent head tilt toward the affected side. No consensus exists regarding the cause of this disorder. In this study, we analyzed various clinical factors in patients with CMT who were treated with surgical release. This analysis enabled us to identify potential causative factors of CMT and to establish a basis for surgical interventions.

Methods In total, 584 patients who underwent surgical intervention for CMT from October 2007 to December 2016 were included in this study. Their demographic characteristics, birthrelated factors, and clinical features were analyzed.

Results Data from 525 patients were analyzed in this study after exclusion of those with insufficient information. Before birth, 31 patients (5.9\%) were diagnosed with oligohydramnios, and $87(16.6 \%)$ had a breech presentation. Seven (1.3\%) cases of clavicle fracture and two $(0.4 \%)$ cases of cephalohematoma were noted at birth. Before surgery, 397 patients (75.6\%) underwent physiotherapy and 128 patients $(24.4 \%)$ did not. The duration of physiotherapy ranged from 1 to 50 months (average, 6 months).

Conclusions Our study shows that $16.6 \%$ of the CMT patients presented in the breech position, which is a much higher rate than that observed in the general population $(3 \%-4 \%)$. We hypothesize that being in the breech position as a fetus appears to exert a significant influence on shortening and fibrosis of the sternocleidomastoid muscle.

Keywords Torticollis / Surgical release / Retrospective studies

\author{
Correspondence: Myong Chul Park \\ Department of Plastic and \\ Reconstructive Surgery, Ajou \\ University Hospital, 164 World cup- \\ ro, Yeongtong-gu, Suwon 16499, \\ Korea \\ Tel: +82-31-219-5614 \\ Fax: +82-31-219-5610 \\ E-mail:mpark0601@gmail.com
}

\section{INTRODUCTION}

Congenital muscular torticollis (CMT) is among the most common musculoskeletal deformities in children and is characterized by a persistent head tilt toward the affected side, with the chin rotated toward the opposite direction [1]. The prevalence of CMT in neonates has historically ranged from $0.3 \%$ to $2.0 \%$ $[1,2]$. Although the clinical features of CMT are well known, no consensus exists regarding the cause of this disorder. Possible etiologies include birth trauma, intrauterine malpositioning, a hereditary component, neurogenic disorders, infection, and sequelae of intrauterine or perinatal compartment syndrome [3-5]. The treatment strategies used for CMT include observation, orthosis application, an active home exercise program, stretching exercises, Botox injections, manual myotomy, and various surgical procedures $[3,6]$. Stretching exercises are sufficient for 
primary treatment in approximately $90 \%$ of cases, while surgical resection of the affected sternocleidomastoid muscle (SCM) is recommended for resistant cases (i.e., no response or improvement after at least 6 months of physiotherapy) [3,7].

In this study, we analyzed various clinical factors in patients with CMT who were treated with surgical resection. Numerous studies have explored the causes of CMT, but no definitive causes have been established. Therefore, we investigated the cause of CMT in patients with severe CMT that required surgery, and sought to identify different possible causes of CMT from those that were proposed in previous studies.

\section{METHODS}

\section{Patients}

In total, 584 patients who underwent surgical intervention for CMT from October 2007 to December 2016 were included in this study. The indications for surgery were over $10^{\circ}$ deficits in passive neck rotation and lateral flexion of the neck for more than 6 months despite physiotherapy. Additionally, palpation of a hard band on (passive) lateral flexion to the contralateral side was personally used as a diagnostic criterion by the senior author. Age over 3 years should be strongly considered as an important factor in the decision regarding whether surgery is indicated. Surgery performed later than 3 to 4 years of age cannot guarantee symmetric growth of the face [8]. The demographic characteristics studied were sex and birth order. The birth-related factors studied were gestational age, multifetal pregnancy, birth weight, delivery method, presence of dystocia, presentation of the fetus, amount of amniotic fluid, health status during pregnancy, perinatal disease, and complications related to birth.
The clinical features of CMT studied were age at the time of diagnosis and surgery, reoperation, the presence of a bulky specimen, age at the beginning of physiotherapy, and total treatment time. We reviewed patients' medical charts to collect these data and interviewed the patients or their guardians to obtain missing data.

\section{Surgical technique}

A single surgeon performed the surgical procedures, which involved distal resection. Monopolar resection was conducted in most cases, while in very few cases, bipolar resection or muscle lengthening was conducted. A skin incision was made transversely 1 to $2 \mathrm{~cm}$ above the clavicle of the affected side. After protecting the external jugular vein, the adjacent fascia and soft tissue were resected. The clavicular head and sternal head of the SCM were exposed, and an approximately $2-\mathrm{cm}$ bulky specimen of the SCM including the fibrotic band was removed (Figs. $1,2)$. If the patient had any limitations in neck range of motion (ROM), the deep fascia and remaining contracted soft tissue were removed at the same time [9]. Acellular dermal matrix was implanted to prevent scar contracture in patients with recurrent CMT or in whom wide resection was conducted due to severe fibrous changes $[9,10]$. A closed suction drain was inserted, and the wound was stitched closed using 5-0 polydioxanone monofilament absorbable suture (PDS; Ethicon, Cornelia, GA, USA) for the subcutaneous and dermis layers; Steri-Strips (Nexcare; $3 \mathrm{M}$, Maplewood, MN, USA) were applied for skin approximation. We changed the wound dressing daily and examined the wound to identify signs of infection, hematoma formation, or any other postoperative complications. When the drainage volume was $<10 \mathrm{~mL} /$ day, the drain was removed. After the surgi-

\section{Fig. 1. View of surgical release}

(A) By exposing the clavicular head and sternal head of the sternocleidomastoid muscle (SCM) and (B) a bulky specimen of the SCM including the fibrotic band was resected.
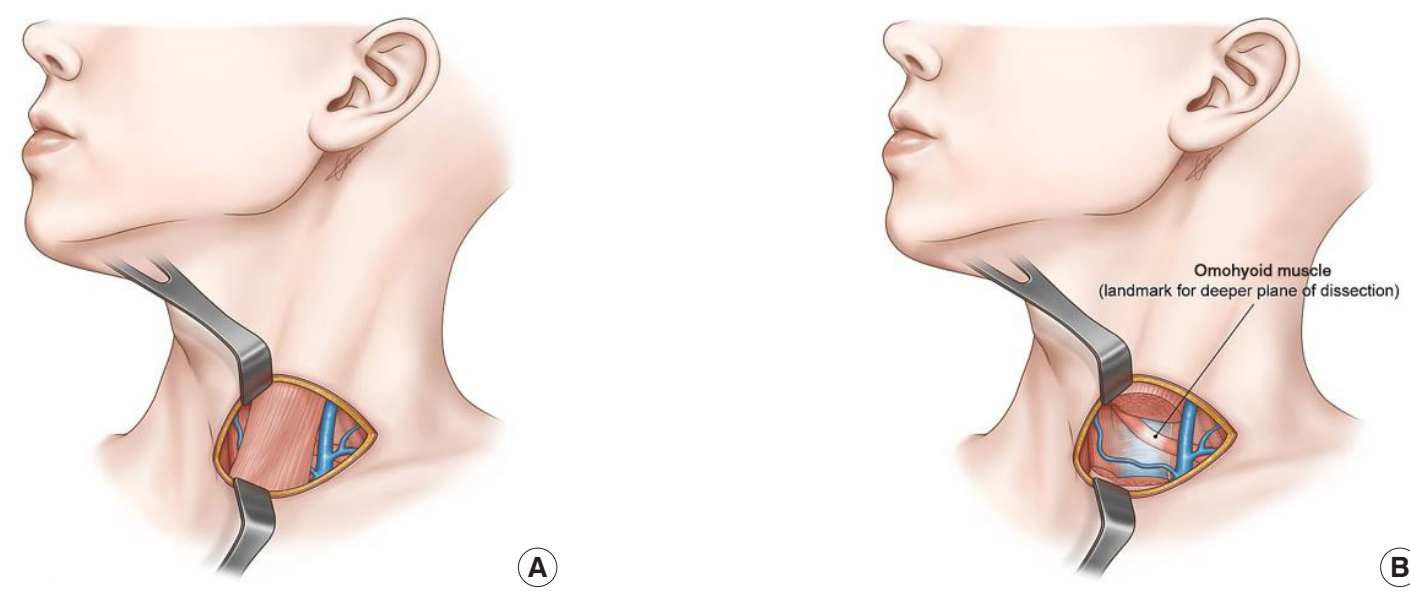


\section{Fig. 2. Intraoperative views}

(A) Surgical design was made before the surgery by touching palpable mass or fibrous band. (B) The contractured soft tissue was completely released by excising a 5 -cm wide fibrous band.

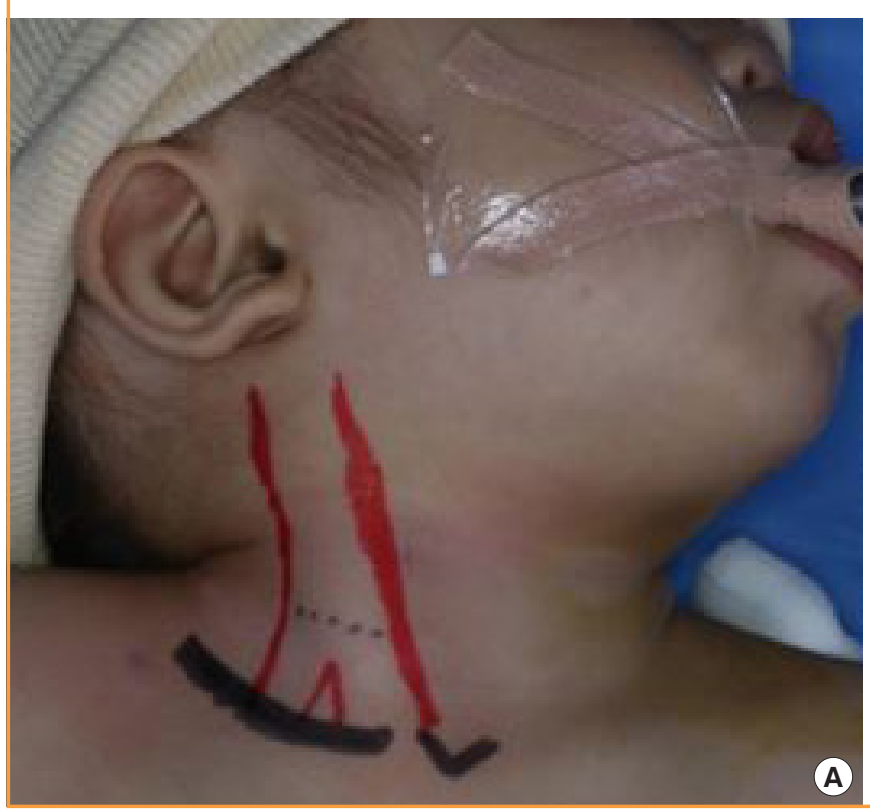

cal procedure, the patients wore a neck collar for 3 weeks and performed stretching exercises for 6 months.

\section{RESULTS}

We reviewed a total of 584 patients, of whom 525 were included in this study, while 59 patients with insufficient available information were excluded.

\section{Demographic characteristics}

Of the 525 patients, 303 were male and 222 were female. One patient $(0.2 \%)$ was a multiplet. With respect to birth order, 453 patients $(86.3 \%)$ were the first child, 62 (11.8\%) were the second child, and 10 (1.9\%) were the third or later child.

\section{Pregnancy- and birth-related issues}

Before birth, 31 patients (5.9\%) had been diagnosed with oligohydramnios. With respect to presentation of the fetus, 437 patients $(83.2 \%)$ had a vertex presentation, 87 patients $(16.6 \%)$ had a breech presentation, and one patient $(0.2 \%)$ had a transverse presentation. In terms of the type of delivery, a normal spontaneous vaginal delivery occurred for 320 patients (61.0\%), labor was induced in 39 patients (7.4\%), forceps delivery and vacuum extraction were performed in 32 patients $(6.1 \%)$, and cesarean delivery occurred in 134 patients (25.5\%) (Table 1). Among the 87 patients in the breech position, 83 underwent a cesarean delivery due to breech presentation. Ninety-seven pa-

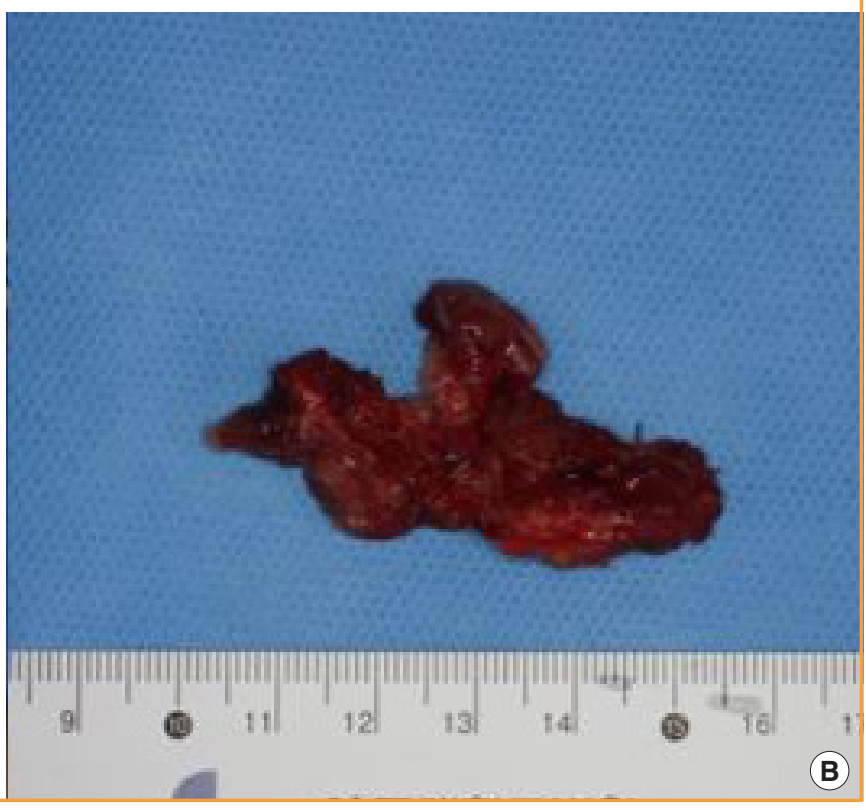

\begin{tabular}{|c|c|}
\hline Variable & No. (\%) \\
\hline \multicolumn{2}{|l|}{ Presentation } \\
\hline Oligohydramnios & 31 (5.9) \\
\hline Vertex & 437 (83.2) \\
\hline Breech & 87 (16.6) \\
\hline Transverse & $1(0.2)$ \\
\hline \multicolumn{2}{|l|}{ Type of delivery } \\
\hline NSVD & $320(61.0)$ \\
\hline Induction of labor & 39 (7.4) \\
\hline FD\&VE & $32(6.1)$ \\
\hline Cesarean delivery & 134 (25.5) \\
\hline
\end{tabular}

tients $(18.3 \%)$ were delivered with dystocia. The average gestational age was 39.3 weeks (range, 33-42 weeks); preterm birth at $<37$ weeks occurred in 13 patients $(2.5 \%)$ and post-term birth at $>42$ weeks occurred in five patients (1.0\%). Patients' birth weight ranged from 1,840 to $4,500 \mathrm{~g}$, and the average birth weight was 3,253.8 g. Four hundred and ninety-five patients (94.3\%) were normal birth weight infant (Table 2). With respect to birth-related issues, meconium aspiration syndrome occurred in eight patients, neonatal asphyxia in eight patients, neonatal jaundice in seven patients, clavicle fracture in seven patients, cephalohematoma in two patients, and neonatal seizure in one patient (Table 3 ). 
Table 2. Birth weight of patients with surgically corrected CMT

\begin{tabular}{|lc|}
\hline Birth weight & No. (\%) \\
\hline ELBWI $(<1,000 \mathrm{~g})$ and VLBWI $(<1,500 \mathrm{~g})$ & 0 \\
LBWI $(\geq 1,500 \mathrm{~g}$ and $<2,500 \mathrm{~g})$ & $13(2.5)$ \\
$\operatorname{NBWI}(\geq 2,500 \mathrm{~g}$ and $\leq 4,000 \mathrm{~g})$ & $495(94.3)$ \\
Large baby $(>4,000 \mathrm{~g})$ & $17(3.2)$ \\
\hline CMT, congenital muscular torticollis; ELBWI, extremely low birth weight infant; \\
VLBWI, very low birth weight infant; LBWI, low birth weight infant; NBWI, normal \\
birth weight infant.
\end{tabular}

\section{Table 3. Incidence of possible causative factors of CMT}

\begin{tabular}{|lcc|}
\hline Causative factor & Normal population (\%) & Our data (\%) \\
\hline Oligohydramnios & $1-5$ & 5.9 \\
Abnormal fetal presentation & & \\
$\quad$ Breech & $3-4$ & 16.6 \\
$\quad$ Transverse lie & 0.3 & 0.2 \\
Birth trauma & & \\
$\quad$ Clavicle fracture & $0.5-1.6$ & 1.3 \\
$\quad$ Cephalohematoma & $1-2$ & 0.4 \\
\hline CMT, congenital muscular torticollis. & & \\
\end{tabular}

\section{Clinical features of CMT}

Among the 525 patients, primary surgery was performed in 509 patients and reoperation in 16 patients. Sixteen patients had an insufficient resection after primary surgery or recurrence of symptoms. Thirteen of those 16 patients underwent primary surgery at other clinics, while the other three patients underwent surgery at our clinic. Approximately $93 \%$ of patients were first diagnosed with CMT at an age $<1$ year (Table 4$)$. At the time of diagnosis, 424 patients $(80.8 \%$ ) had a bulky specimen, and 101 had no bulky specimen or did not know whether they had a bulky specimen. The surgical operations were performed from age 0 to 41 years (average age, 4.4 years). The number and proportion of patients in each age group are shown in Table 5.

In $51 \%$ of patients, the duration from diagnosis to surgery was $<1$ year. Before surgery, 397 patients underwent physiotherapy and 128 patients did not. The duration of physiotherapy ranged from 1 to 50 months (average, 6 months) (Table 6).

\section{DISCUSSION}

CMT is defined as unilateral shortening or contracture of the SCM caused by fibrosis of unknown cause in newborn infants or young children. The shortening of the SCM on the involved side results in an ipsilateral head tilt and contralateral rotation of the chin. This limits head mobility in both rotation toward the affected side and lateral flexion toward the other side. Although several theoretical proposals have been made, uncertainties re-
Table 4. Surgically corrected CMT by age at diagnosis

\begin{tabular}{|lc|}
\hline Diagnosis date & No. (\%) \\
\hline Under the age of 1 yr & $429(81.7)$ \\
Age 1 yr & $19(3.6)$ \\
Age 2 yr & $8(1.5)$ \\
Age 3 yr & $10(1.9)$ \\
Age 4 yr & $13(2.4)$ \\
Age 5 yr & $6(1.1)$ \\
Age 6 yr & $5(0.9)$ \\
Age 7 yr & $8(1.5)$ \\
Age 8 yr & $6(1.1)$ \\
Age 9 yr & 0 \\
Age 10 yr & $6(1.1)$ \\
Over the age of 10 yr & $15(2.8)$ \\
\hline CMT, congenital muscular torticollis. & \\
\hline
\end{tabular}

Table 5. Surgically corrected CMT by age at the operation

\begin{tabular}{|lc|}
\hline Age at the operation & No. (\%) \\
\hline Under the age of $1 \mathrm{yr}$ & $110(21.0)$ \\
Age $1-4 \mathrm{yr}$ & $236(45.0)$ \\
Age $5-10 \mathrm{yr}$ & $127(24.2)$ \\
Age 11-20 yr & $37(7.0)$ \\
Over the age of $20 \mathrm{yr}$ & $15(2.9)$ \\
\hline CMT, congenital muscular torticollis. & \\
\hline
\end{tabular}

Table 6. Surgically corrected CMT by duration of physiotherapy

\begin{tabular}{|lr|}
\hline Duration of physiotherapy & No. (\%) \\
\hline Under 3 mon & $188(35.8)$ \\
3-6 mon & $104(19.8)$ \\
6-12 mon & $151(28.8)$ \\
Over 12 mon & $82(15.6)$ \\
\hline CMT, congenital muscular torticollis. \\
\hline
\end{tabular}

main regarding the actual etiology of CMT [11].

Many studies have been performed to identify the causes of CMT. An abnormal fetal presentation and resultant birth trauma were initially considered to be the cause of CMT. Abnormal presentations include breech presentation and transverse lie presentation. Breech presentation is characterized by entrance of the fetus's buttock or feet into the pelvis. Transverse lie presentation is characterized by orientation of the fetal shoulder towards the maternal pelvis. Birth trauma was historically believed to result in hematoma formation in the SCM, leading to fibrous contracture of the muscle [12]. However, this theory does not explain the occurrence of CMT after cesarean sections $[4,13]$. Later suggestions include hereditary factors, neurogenic disorders, infection, and sequelae of intrauterine or perinatal compartment syndrome $[5,11]$. However, these hypotheses were 
mainly studied from the 1930s to 1950s. The most recent case report advocating for the hereditary hypothesis was published in 1997 [4]. Overall, the etiology of CMT remains unclear.

To the best of our knowledge, the actual incidence of oligohydramnios, abnormal fetal presentation, and birth-related injury has not been established. The reported rates of oligohydramnios in the general population are influenced by inconsistencies in diagnostic criteria, the population analyzed, the threshold used, and the gestational age at the time of the ultrasound examination [14]. One study reported that oligohydramnios (defined as an amniotic fluid index of $\leq 5 \mathrm{~cm}$ ) occurs in about $1 \%-5 \%$ of pregnancies at term [14]. In our study group, oligohydramnios was noted in $5.9 \%$ of patients. Considering the incidence of oligohydramnios in the general population, the incidence of oligohydramnios among the patients with CMT in our study was rather high. Reported rates of abnormal fetal presentation are also influenced by variations in the population studied and the gestational age at the time of the ultrasound examination. Generally, $3 \%-4 \%$ of fetuses are breech at term, and approximately 1 in 300 fetuses has a transverse presentation at delivery [15-18]. The rates of abnormal fetal presentations in our study group were $16.6 \%$ for a breech presentation and $0.2 \%$ for a transverse presentation, which are markedly higher than the reported rates in the general population. The overall incidence of birth injuries is roughly $2.0 \%$ and $1.1 \%$ in singleton vaginal deliveries of fetuses in a cephalic position and in cesarean deliveries, respectively $[19,20]$. In the present study, clavicle fractures and cephalohematomas were reported in $1.3 \%$ and $0.4 \%$ of patients, respectively. Based on data obtained from large case series, the incidence of clavicle fractures due to birth trauma ranges from $0.5 \%$ to $1.6 \%$ [21-23]. Cephalohematoma is estimated to occur in $1 \%-2 \%$ of all deliveries [19]. Although some limitations prevent a direct comparison of the reported incidence in this study with that in the general population, we can suggest that the incidence of several factors potentially associated with torticollis during the past few decades was not particularly high (e.g., oligohydramnios and birth injury). However, $16.6 \%$ of the patients with CMT were in the breech position; this is a much higher rate than that observed in the general population $(3 \%-4 \%)$. Therefore, we can hypothesize that the breech position seems to exert a significant influence on shortening and fibrosis of the SCM.

Furthermore, in some cases, patients who were not reported to have experienced birth trauma had bony hypertrophy or severe tissue adhesion, which is usually seen on the fracture site of clavicle after healing. It is possible that those patients might have experienced birth trauma, such as clavicle fractures, but the parents were not aware of the birth trauma when we interviewed them. This consideration suggests that the incidence of birth trauma could have been underestimated, and this possibility should be investigated in further studies.

In our study, the bulky specimen, which patients' parents usually referred to as a "mass," was seen in the form of an asymmetric marked thickening and internal low signal alteration on magnetic resonance imaging. Fibromatosis can also be confirmed by biopsy. Patients with CMT can benefit from a surgical intervention when a bulky specimen or fibrous band on the neck is palpated with the head tilted toward the affected side, shortening of the ipsilateral SCM is detected, or deficits are found in passive neck rotation or lateral flexion. Because age at the time of surgery is believed to be the most important factor influencing the outcome of surgery and complications of resistant CMT, surgical treatment is recommended at 1-4 years of age [24]. For various reasons, however, some patients with CMT do not undergo surgical treatment despite meeting the clinical criteria. In the present study, $66.0 \%$ of patients underwent a surgical intervention before the age of 4 years, $24.2 \%$ between the age of 5 and 10 years, $7.0 \%$ between the age of 11 and 20 years, and $2.9 \%$ after the age of 20 years. Additionally, surgical resection of the affected SCM is recommended when no response or improvement is seen after at least 6 months of physiotherapy [3,7]. In our study, the average duration of preoperative rehabilitation was 6 months; however, some patients underwent surgery after more than 8 years of rehabilitation. There are many possible reasons for delayed surgery. The patient's guardian may refuse the invasive treatment, and the clinician or guardian may not be aware that a surgical intervention can be helpful if the patient's symptoms do not improve after at least 6 months of physiotherapy. After birth, if a guardian tries to tilt the baby's neck and a bulky specimen of the neck is suspected, the guardian usually starts by visiting the rehabilitation medicine department, and then physiotherapy is performed first if a diagnosis is made. Patients who initially had a bulky specimen or a difference of more than $20^{\circ}$ at the initial visit to the plastic surgeon, rather than in their visit to the rehabilitation medicine department, proceeded to surgery without any physiotherapy because physiotherapy results in minimal improvements in such patients. The variation in the duration of physiotherapy reflects differences in when the rehabilitation department transfers the care of the patient to the plastic surgery department.

Our experience indicates that surgical treatment for neglected CMT effectively achieves a satisfactory ROM of the neck and improves secondary skeletal deformities, including spinal deformities. Our center previously reported favorable results in patients older than 8 years old with recurrent or neglected CMT who underwent conventional unipolar resection with or without acellular dermal matrix grafting [9]. Therefore, clinicians 
should not hesitate to recommend surgical treatment for neglected CMT in older patients.

There are several limitations of this study. We directly compared the results of this study with data in the general population. Additionally, the results include several factors that may be influenced by the development and accessibility of the healthcare system. Because some patients' medical records were lacking, we had to obtain additional information by interviewing the patients or their guardians, which may have reduced the accuracy of the information. Additionally, we only investigated patients who had undergone surgery. Considering that $90 \%$ of patients are treated primarily through stretching exercises, the results of this study are not representative of all patients with CMT.

Although the exact etiology of CMT is still unknown, we identified the incidence of several possible causes of torticollis. The incidence of oligohydramnios and birth injury was not remarkably high. However, because fetal malpositioning, especially the breech position, can cause torticollis, more studies are needed on this cause-and-effect relationship based on ultrasound examinations of fetuses in the breech position. Whether the position of the tilted head of the fetus in utero is associated with the later occurrence of torticollis on the affected side also remains unclear. This study also revealed that many patients with CMT requiring a surgical intervention missed the optimal timing for surgery after a relatively long duration of rehabilitation. Clinicians and patients require more consultations and education about the treatment schedule of CMT.

\section{NOTES}

\section{Conflict of interest}

No potential conflict of interest relevant to this article was reported.

\section{Ethical approval}

The study was approved by the Institutional Review Board of Ajou Medical Center (IRB No. AJIRB-MED-MDB-19-064) and performed in accordance with the principles of the Declaration of Helsinki. Written informed consents were obtained.

\section{Patient consent}

The patient's parent provided written informed consent for the publication and the use of patient images.

\section{Author contribution}

Conceptualization: Park MC. Data curation: Jeong KS. Formal analysis: Ha NH. Methodology: Cha B. Project administration: Kim SM. Visualization: Cha B. Writing - original draft: Kim SM.
Writing - review \& editing: Park MC. Approval of final manuscript: all authors.

\section{ORCID}

Sue Min Kim https://orcid.org/0000-0002-0894-5207

Bohwan Cha https://orcid.org/0000-0002-4780-6525

Kwang Sik Jeong https://orcid.org/0000-0002-0687-0862

Non Hyeon Ha https://orcid.org/0000-0003-1797-1834

Myong Chul Park https://orcid.org/0000-0002-0845-2672

\section{REFERENCES}

1. Suhr MC, Oledzka M. Considerations and intervention in congenital muscular torticollis. Curr Opin Pediatr 2015;27: 75-81.

2. Do TT. Congenital muscular torticollis: current concepts and review of treatment. Curr Opin Pediatr 2006;18:26-9.

3. Cheng JC, Tang SP, Chen TM, et al. The clinical presentation and outcome of treatment of congenital muscular torticollis in infants: a study of 1,086 cases. J Pediatr Surg 2000; 35:1091-6.

4. Engin C, Yavuz SS, Sahin FI. Congenital muscular torticollis: is heredity a possible factor in a family with five torticollis patients in three generations? Plast Reconstr Surg 1997; 99:1147-50.

5. Davids JR, Wenger DR, Mubarak SJ. Congenital muscular torticollis: sequela of intrauterine or perinatal compartment syndrome. J Pediatr Orthop 1993;13:141-7.

6. Joyce MB, de Chalain TM. Treatment of recalcitrant idiopathic muscular torticollis in infants with botulinum toxin type a.J Craniofac Surg 2005;16:321-7.

7. Cheng JC, Wong MW, Tang SP, et al. Clinical determinants of the outcome of manual stretching in the treatment of congenital muscular torticollis in infants: a prospective study of eight hundred and twenty-one cases. J Bone Joint Surg Am 2001;83:679-87.

8. Seo SJ, Kim JH, Joh YH, et al. Change of facial asymmetry in patients with congenital muscular torticollis after surgical release. J Craniofac Surg 2016;27:64-9.

9. Hahn HM, Cook KH, Lee IJ, et al. Use of acellular dermal matrix in treatment of congenital muscular torticollis in patients over eight years of age. J Craniofac Surg 2017;28:6105.

10. Lee IJ, Lim SY, Song HS, et al. Complete tight fibrous band release and resection in congenital muscular torticollis. J Plast Reconstr Aesthet Surg 2010;63:947-53.

11. Tatli B, Aydinli N, Caliskan M, et al. Congenital muscular torticollis: evaluation and classification. Pediatr Neurol 2006; 
34:41-4.

12. Whitman R. Observations on torticollis, with particular reference to the significance of the so-called haematoma of the sterno-mastoid muscle. J Bone Joint Surg 1891;4:293-307.

13. Wolfort FG, Kanter MA, Miller LB. Torticollis. Plast Reconstr Surg 1989;84:682-92.

14. Moore TR. Clinical assessment of amniotic fluid. Clin Obstet Gynecol 1997;40:303-13.

15. Gemer O, Segal S. Incidence and contribution of predisposing factors to transverse lie presentation. Int J Gynaecol Obstet 1994;44:219-21.

16. Cruikshank DP, White CA. Obstetric malpresentations: twenty years' experience. Am J Obstet Gynecol 1973;116:1097104.

17. Scheer K, Nubar J. Variation of fetal presentation with gestational age. Am J Obstet Gynecol 1976;125:269-70.

18. Hickok DE, Gordon DC, Milberg JA, et al. The frequency of breech presentation by gestational age at birth: a large pop- ulation-based study. Am J Obstet Gynecol 1992;166:851-2.

19. Alexander JM, Leveno KJ, Hauth J, et al. Fetal injury associated with cesarean delivery. Obstet Gynecol 2006;108:88590.

20. Demissie K, Rhoads GG, Smulian JC, et al. Operative vaginal delivery and neonatal and infant adverse outcomes: population based retrospective analysis. BMJ 2004;329:24-9.

21. Beall MH, Ross MG. Clavicle fracture in labor: risk factors and associated morbidities. J Perinatol 2001;21:513-5.

22. Hsu TY, Hung FC, Lu YJ, et al. Neonatal clavicular fracture: clinical analysis of incidence, predisposing factors, diagnosis, and outcome. Am J Perinatol 2002;19:17-21.

23. Lam MH, Wong GY, Lao TT. Reappraisal of neonatal clavicular fracture: relationship between infant size and neonatal morbidity. Obstet Gynecol 2002;100:115-9.

24. Ling CM. The influence of age on the results of open sternomastoid tenotomy in muscular torticollis. Clin Orthop Relat Res 1976;(116):142-8. 